\title{
Review on Chemistry and Bioactivities of Secondary Metabolites from Some Medicinal Plants and Microbes of Bangladesh
}

\author{
Mohammad A. Rashid, Mohammad R. Haque, Md. Al Amin Sikder, \\ Abu A. Chowdhury, Mohammad S. Rahman and Choudhury M. Hasan \\ Phytochemical Research Laboratory, Department of Pharmaceutical Chemistry, \\ Faculty of Pharmacy, University of Dhaka, Dhaka-1000, Bangladesh
}

\begin{abstract}
Plants and microorganisms, being the major source of many drugs, have attracted scientists from ancient times. However, until recently an insignificant part of the plants and some microorganisms have scientifically been evaluated for their medicinal values. The present study was undertaken to discover new drug candidates from natural sources. Extensive chemical studies with 60 medicinal plants and several microbial strains of Bangladesh have resulted in the isolation and characterization of 150 compounds, including 50 new molecules. Terpenoids and alkaloids were the major constituents among the isolated compounds. The crude extractives and several purified molecules demonstrated statistically significant inhibition of growth of microorganisms, antioxidant, antidiabetic and HIV-inhibitory activities. Usnic acid, a lead compound isolated from the lichen, Parmelia kamtschandalis, showed potent antimicrobial activity, whereas dehydroaltenusin obtained from a Streptomyces sp. exhibited significant HIV-inhibitory effects.

Keywords: Medicinal Plants, secondary metabolites, antioxidant, antidiabetic, HIV-inhibitory activity.
\end{abstract}

\section{Introduction}

Medicinal plants are the blessings for any country which contribute a lot for traditional health management as well as providing lead compounds for modern drug discovery. The varieties of molecules contained in plants have been proved to combat complicated diseases. Based on this, natural product scientists have always focused on the isolation of bioactive compounds from these precious herbs and trees. In addition, the giant pharmaceutical companies are also capitalizing these scopes for incorporating new drugs in the market (Burnett et al., 2012; Christen and Cuendet, 2012; Hung et al., 2012; Lovkova et al., 2001 and Newman and Cragg, 2012).

Bangladesh being a subtropical country is a good repository of plants. There are around 5000 angiosperms distributed among 200 families. Approximately, 500 of these are being used in the traditional medicines for the treatment of different types of diseases. A significant percentage of the population depends on the natural product based medicines. In addition, the total medicinal plant market of Bangladesh is equivalent to US\$14 billion each year. As part of our continuing studies on plants here, we summarize the chemistry and bioactivities of some of the isolated constituents from 48 medicinal plants of Bangladesh (Ara et al., 2006; Begum et al., 2011; Islam et al., 2009; Jahan et al., 2009 and Rahman et al., 2011).

\section{Materials and Methods}

Chemical: The chemical investigation of a plant involved collection and prop er identification of the plant materials, extraction, fractionation and purification of compounds and structural characterization of the purified secondary metabolites. On the other hand, the culture filtrate of the microorganism's broth culture was extracted with ethyl acetate. Various chromatographic techniques (Zhu et al., 2003; Moustafa et al., 2007; Widodo et al., 2008 and Jain and Bari, 2010) were utilized for isolation and purification of the compounds from the extractives. The structures of the purified compounds were determined by extensive analyses of UV, IR, NMR and mass spectroscopic data as well as by chemical derivatization, when needed. Whenever possible, the crude extracts, fractions and purified compounds were subjected to bioassays (e.g.

Correspondence to: Mohammad A. Rashid; Tel.: 880-2-9661900, Extn. 8137; Fax: 880-2-8615583; E-mail: rashidma@du.ac.bd 
antimicrobial activity, antioxidant, antidiabetic, HIV inhibitory activities etc.) The whole process can be explained by scheme 1 .

Antimicrobial activity: The antimicrobial activity of the purified compounds (Bhilabutra et al., 2007; Ahamed et al., 2007 and Ghani et al., 2012) was determined by the disc diffusion method (Sunilson et al., 2009). The bacterial strains were collected as pure cultures from the Institute of Nutrition and Food Science (INFS), University of Dhaka, Bangladesh. The samples were dissolved separately in chloroform and applied to sterile discs at 100 or $30 \mu \mathrm{g} /$ disc and carefully dried to evaporate the residual solvent. Here, kanamycin, amoxicillin, streptomycin and tetracycline were used as standard antimicrobial agents.

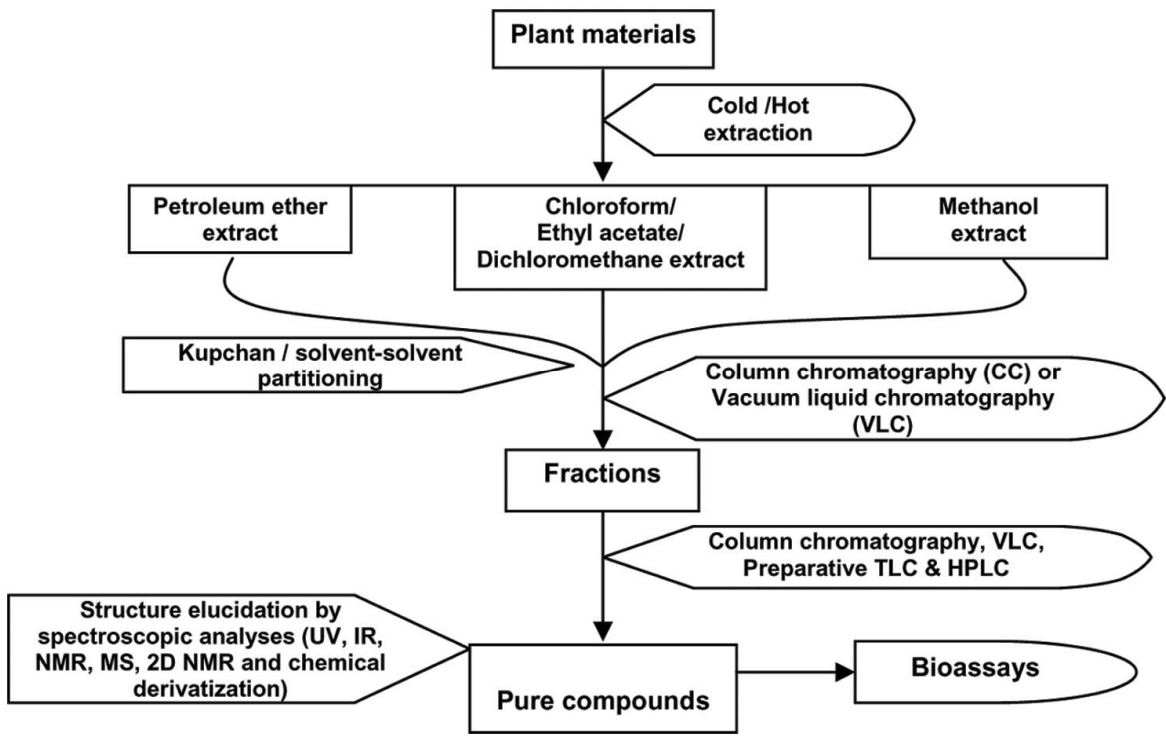

Scheme 1. Isolation, purification and bioassay of compounds.

Antioxidant activity: The antioxidant (free radical scavenging) activity of the compounds was assessed by the method of Brand-Williams (Brand-Williams et al., 1995; Aher et al., 2009 and Ham et al., 2010). Percentage inhibitions were plotted against respective concentrations used and from the graph obtained, the $\mathrm{IC}_{50}$ was calculated. Tert-butyl-1-hydroxytoluene (BHT), a potential antioxidant, was used as positive control.

Antidiabetic activity: Antidabetic activity was investigated on alloxan-induced Long Evan's rats following the procedure published elsewhere (Mansour et al., 2002). The rats (weighing 100-200 g were used for the study) were obtained from international Centre for Diarrheal Disease Research, Bangladesh (ICDDR,B), Dhaka. The experimental procedure is shown in Table 1.

Anti-HIV assay: The purified compound was dissolved in DMSO, diluted to the desired concentration and tested in a XTT-based in vitro antiHIV assay (Gulakowski et al., 1991).
Table 1. Design of the experiment for antidiabetic study.

\begin{tabular}{|c|c|c|}
\hline & Phase-1 & Phase-2 \\
\hline $\begin{array}{l}\text { Test } \\
\text { materials }\end{array}$ & $\begin{array}{l}\text { Methanol extract } \\
\text { of whole plant }\end{array}$ & Eclalbasaponin II \\
\hline Duration & 4 weeks & $\begin{array}{l}1 \text { week (due to lack } \\
\text { of sample) }\end{array}$ \\
\hline \multicolumn{3}{|l|}{ Group of rats } \\
\hline$\overline{\mathrm{Gr}-1}$ & Normal untreated & Normal untreated \\
\hline Gr-2 & Alloxan treated & Alloxan treated \\
\hline $\mathrm{Gr}-3$ & $\begin{array}{l}\text { Glibenclamide } \\
\text { treated }\end{array}$ & $\begin{array}{l}\text { Glibenclamide } \\
\text { treated }\end{array}$ \\
\hline & (600 $\mu \mathrm{g} / \mathrm{kg}$ bw orally) & $\begin{array}{l}(600 \mu \mathrm{g} / \mathrm{kg} \text { bw } \\
\text { orally) }\end{array}$ \\
\hline Gr-4 & $\begin{array}{l}\text { Plant extract treated } \\
(300 \mathrm{mg} / \mathrm{kg} \text { bw } \\
\text { orally) }\end{array}$ & $\begin{array}{l}\text { eclalbasaponin II } \\
\text { treated orally } \\
(10 \mathrm{mg} / \mathrm{kg} \mathrm{bw} \\
\text { orally) }\end{array}$ \\
\hline \multicolumn{3}{|l|}{ Analysis } \\
\hline Body weight & At weekly interval & After a week \\
\hline Blood sugar & $\begin{array}{l}\text { At weekly interval } \\
\text { for } 28 \text { days }\end{array}$ & $\begin{array}{l}\text { At two days interval } \\
\text { for } 7 \text { days }\end{array}$ \\
\hline $\begin{array}{l}\text { Hepato- } \\
\text { toxicity }\end{array}$ & $\begin{array}{l}\text { ALT, AST and ALP } \\
\text { at } 28 \text { th day }\end{array}$ & Not done \\
\hline
\end{tabular}

\section{Results}

Chemical: Extensive chromatographic separation and purification of the extracts obtained from 48 
medicinal plants of Bangladesh afforded a total of 150 pure chemical entities, including 37 new molecules (Rahman et al., 2001). The structures of these compounds were elucidated by extensive spectroscopic studies including 2D NMR and MS and chemical derivatization wherever needed. The structures of some of the isolated compounds are shown below:

Sesquiterpenes from Amoora rohituka Roxb. (Meliaceae) (Chowdhury et al., 2003)<smiles>CC1=C2C3OC3(C(C)C)CCC(C)[C@@H]2CC1=O</smiles>

6 $\beta, 7 \beta$-Epoxyguai-4-en-3-one<smiles>CC(C)[C@]12CC[C@@H](C)[C@H]3CC[C@](C)(O)C3(O)C1O2</smiles>

$6 \beta, 7 \beta$-Epoxy-4 $\beta, 5$-dihydroxyguaiane

Triterpenes and phenylpropanoid from Amoora cucullata Roxb. (Meliaceae) (Rahman et al., 2005), Corypha taliera Roxb. (Palmae) (Chowdhury et al., 2013) and Mesua nagassarium Burm.f.

(Clusiaceae) (Islam, 2012)<smiles>C[C@H]1C(=O)CC[C@H]2[C@@]3(C)CC[C@H]4C5CC(C)(C)CC[C@]5(C)CC[C@]4(C)C3CC[C@@]21C</smiles>

Fridelin<smiles>O=C(O)/C=C/c1ccc(O)c(O)c1</smiles>

Caffeic acid<smiles>C=C(C)[C@H]1CCC2(C(=O)O)CC[C@]3(C)C(CCC4[C@@]5(C)CC[C@H](O)C(C)(C)C5CC[C@]43C)C12</smiles>

Betulinic acid
Terpenoids from Melicope indica Wt. (Rutaceae) (Farruque et al., 2003)<smiles>CC(C)[C@H]1CCC2=C3CCC4C(CCC5C(C)(C)[C@@H](O)CC[C@]45C)[C@@]3(C)CC[C@@]21C</smiles>

Neohop-13(18)-en-3 $\alpha$-ol<smiles>CC(C)[C@H]1CCC2[C@]1(C)CCC1(C)C3=C(CC[C@]21C)[C@@]1(C)CC[C@@H](O)C(C)(C)C1CC3</smiles>

Fern-8(9)-en-3 $\beta$-ol

Steroids from Artabotrys odoratissimus R.Br (Hasan et al., 1987) and B from Desmos longiflorus Roxb.(Connolly et al., 1994) (Annonaceae)<smiles>C=C(CCC(C)C1CC[C@]2(C)C3=CCC4C(C)(C)[C@@H](O)CC[C@]4(C)C3=CC[C@]12C)C(C)C</smiles>

24-Methylene-lanosta-7,9(11)dien-3 $\beta$-ol (A)<smiles>C=C(CCC(C)C1C[C@H](O)[C@]2(C)C3=CCC4C(C)(C)[C@@H](O)CC[C@]4(C)C3=CC[C@]12C)C(C)C</smiles>

$15 \alpha$-Hydroxy-24-methylenelanosta-7,9(11)-dien-3-ol (B)

Diterpene from Polyalthia longifolia var. pendulla

(Annonaceae) (Hasan et al., 1995)<smiles>C=C1CCCC2C(CCC3=CC(=O)O[C@@H]3O)[C@H](C)CC[C@]12C</smiles>

16 $\beta$-Hydroxykolava-4,3Z-dien-15,16-olide 


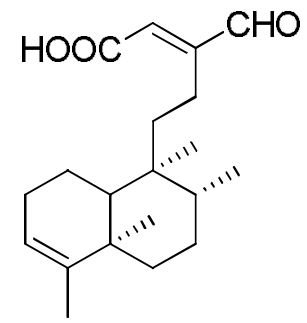

(-)-16-oxocleroda-3,13(14)E-dien-15-oic acid<smiles>CC1=CCCC2[C@]1(C)CC[C@@H](C)[C@]2(C)CCC1=CC(=O)O[C@@H]1O</smiles>

(-)-16 $\alpha$-hydroxycleroda-3,13 (14)Z-dien-15,16-olide

Steroid from Toona ciliata M. Roem (Meliaceae) (Chowdhury et al., 2003)

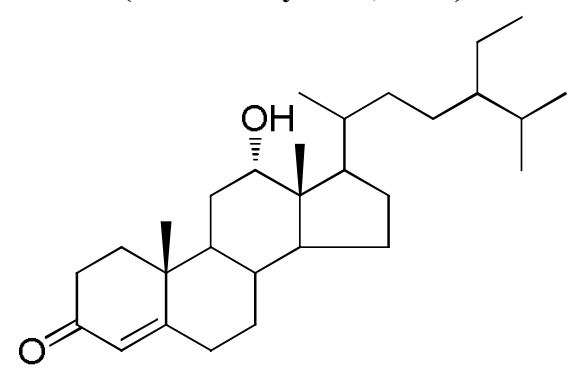

$12 \alpha$-Hydroxystigmast-4-en-3-one

Triterpene glycosides from Corchorus capsualris L. (Tiliaceae) (Hasan et al., 1984; Quader et al., 1987)

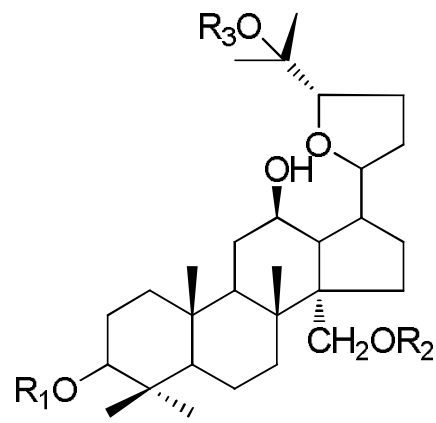

$\mathrm{R}_{1}=\mathrm{R}_{2}=\mathrm{R}_{3}=\mathrm{H}$ : Capsugenine

$\mathrm{R}_{1}=\mathrm{R}_{3}=\mathrm{H}, \mathrm{R}_{2}=$ Glucose: Capsugenine-30-O- $\beta$ glucopyranoside

$\mathrm{R}_{1}=\mathrm{H}, \mathrm{R}_{2}=\mathrm{R}_{3}=$ Glucose: Capsugenine-25, 30-O- $\beta$ glucopyranoside
Steroids from Nerium oleander L. (Apocynaceae)

(Huq et al., 1999a; Huq et al., 1999b)<smiles>[R]C1CC[C@]2(C)C3=C(CCC2C1)C1=CC=C(C2=CC(=O)OC2[R3])[C@]1(C)[C@@H]([R2])C3</smiles>

$\mathrm{R}_{1}=\mathrm{OH}, \quad \mathrm{R}_{2}=\mathrm{R}_{3}=\mathrm{H}:$ 3-Hydroxy-5-carda8,14,16,20(22)-tetraenolide

$\mathrm{R}_{1}=\mathrm{R}_{3}=\mathrm{H}, \quad \mathrm{R}_{2}=\mathrm{OH}:$ 12-Hydroxy-5-carda8,14,16,20(22)-tetraenolide

$\mathrm{R}_{1}=$ glu, $\mathrm{R}_{2}=\mathrm{H}, \mathrm{R}_{3}=\mathrm{OH}:$ 21-Hydroxy-5-carda-

8,14,16,20(22)-tetraenolide-3- $\beta$-digitaloside<smiles>CC(=O)C1=CCC2C3C=CC4=CC(=O)CC[C@]4(C)C3C[C@H](O)[C@]12C</smiles>

Neridienone A

Terpenoids from Polygonum viscosum Buch. (Polygonaceae) (Datta et al., 2002)

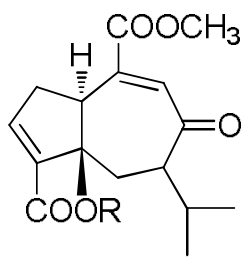

$\mathrm{R}=\mathrm{H}$ : Viscoazusone; $\mathrm{R}=\mathrm{CH}_{3}$ : Viscoazulone

Terpenoids from Coffea bengalensis Roxb. (Rubiaceae) (Hasan et al., 1995)

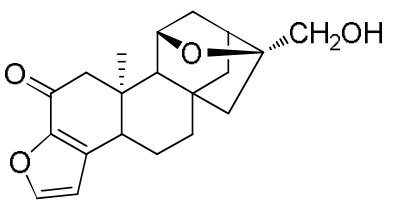

Bengalensol

Diterpenoids from Barringtonia recemosa $\mathbf{L}$. (Lecythidaceae) (Hasan et al., 2000)<smiles>COC(=O)[C@H]1CC[C@]23COC(=O)C2=CCCC3C1CC(=O)c1ccoc1</smiles>

Nasimalun A 
<smiles>COC(=O)C1=CCCC2C(=O)CCC1C2CC(=O)c1ccoc1</smiles>

Nasimalun B

Coumarins and limonoids from Clausena heptaphylla Roxb. (Rutaceae) (Begum et al., 2011; Sohrab et al., 1999)

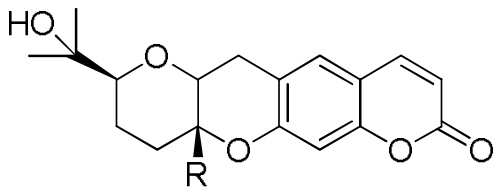

$\mathrm{R}=\mathrm{H}$ : Lunamarin $\mathrm{A}$;

$\mathrm{R}=\mathrm{CH}_{3}$ : Lunamarin $\mathrm{B}$

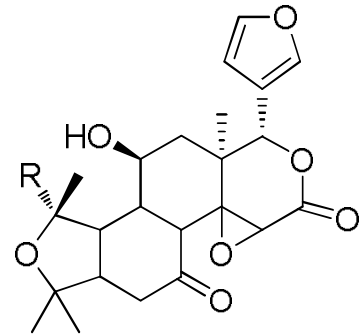

$\mathrm{R}=$ OMe: Clausenolide-1-methyl ether $\mathrm{R}=\mathrm{OH}$ : Clausenolide<smiles>CC(C)(O)C(=O)CC[C@]1(C)Oc2cc3c(cc2C[C@@H]1O)C=CC(=O)C3</smiles>
Lunamarin $\mathrm{C}$<smiles>CC(C)=CCc1c(O)c(C=O)cc2c1[nH]c1c(O)cccc12</smiles>

8-Hydroxyheptaphylline<smiles>CC1(C)OC(=O)C[C@@H](O)C2(C)C1CC(=O)C1(C)C3OC3C(=O)O[C@H](c3ccoc3)[C@@]2(C)C[C@H]1O</smiles>

Clausenarin
Alkaloid and coumarins from Clausena suffruticosa Roxb. (Rutaceae) (Begum et al., 2008)<smiles>COc1c(C=O)cc2c([nH]c3ccccc32)c1CC=C(C)C</smiles>
$O$-Methylheptaphylline<smiles>CC1=CC(C/C(C)=C/COc2ccc3ccc(=O)oc3c2)OC1=O</smiles>

Coumarins and flavonoid from Micromelum minutum G. Forster (Rutaceae) (Sohrab et al., 2004)<smiles>COc1cc2c(cc1C(=O)C1OC1(C)CO)C=CC(=O)C2</smiles>

Hopeyhopol<smiles>CC(C)(O)C1Cc2cc3ccc(=O)oc3cc2O1</smiles>

Marmesin

Flavonoids from Unona discolor/Uvaria chinensis Vahl. (Annonaceae) (Asha et al., 2003)<smiles>[R2]c1c([R7])c(O)c2c(c1[R2])C[C@]([R4])(c1ccccc1)CC2=O</smiles>

$\mathrm{R}_{1}=\mathrm{CH}_{3}, \mathrm{R}_{2}=\mathrm{H}, \mathrm{R}_{3}=\mathrm{CHO}, \mathrm{R}_{4}=\mathrm{H}$ : 8-Formyl-6methyl-5-hydroxyflavanone $\mathrm{R}_{1}=\mathrm{CH}_{3}, \mathrm{R}_{2}=\mathrm{OH}, \mathrm{R}_{3}=\mathrm{CHO}, \mathrm{R}_{4}=\mathrm{OH}$ : 8-Formyl-6methyl-2 $\beta, 5,7$-trihydroxyflavanone $\mathrm{R}_{1}=\mathrm{CHO}, \mathrm{R}_{2}=\mathrm{OH}, \mathrm{R}_{3}=\mathrm{CH}_{3}, \mathrm{R}_{4}=\mathrm{OH}$ : 6-formyl-8Methyl-2 $\beta, 5,7-t r i h y d r o x y f l a v a n o n e$ 
Alkaloid and Acetogenin from Miliusa velutina

(Dunal) Hook. (Annonaceae)(Jumana et al., 2000a;

Jumana et al., 2000b)<smiles>COc1ccc2c(c1O)-c1c(cc(OC)c(OC)c1O)CC2[N+](C)([O-])[O-]</smiles>

(+)-Isocorydine- $\alpha-\mathrm{N}$-oxide<smiles>C=CCCCCCC/C=C\CCCCCCCCCC1CC(C=O)OC1=O</smiles>

Isogoniothalamusin

Coumarins from Murraya koenigii L. (Rutaceae) (Nutun et al., 1999)<smiles>COc1cc2[nH]c3c(C/C=C(\C)CCC=C(C)C)c(O)ccc3c2cc1C</smiles>

Murrayanol<smiles>CCC/C(C)=C/Cc1c(OC)ccc2c1[nH]c1cc(O)c(C)cc12</smiles>

Isomurrayanol<smiles>CC(C)=CCCC1(C)C=Cc2c(ccc3c2[nH]c2cc(O)c(C)cc23)O1</smiles>

Isomahanime<smiles>COc1ccc2c(c1)[nH]c1c(-c3c(O)c(C)cc4c3[nH]c3c(C/C=C(\C)CCC=C(C)C)c(OC)ccc34)c(O)c(C)cc12</smiles>

Bismurrayafoline $\mathrm{E}$
Styryl lactone from Goniothalamus sesquipedalis Wall. (Annonaceae) (Hasan et al., 1994; Hasan et al., 1996)<smiles>CCCCCCCCCCCCCCC(O)C(O)CCC(O)C1CC(C(O)CCCCC(O)CC2=CCOC2=O)C(=O)O1</smiles>

Gigantopentocin<smiles>CC(C)(O)[C@H]1C=CC(=O)OC1[C@H]1O[C@@H]1c1ccccc1</smiles>

5-Acetoxy isogoniothlamineoxide

Anthraquinones and napthaquinones from Stereospermum chelonoides (L.f.) DC (Bignoniaceae) (Haque et al., 2006)<smiles>CC(=O)CCc1ccc2c(c1C)C(=O)c1ccccc1C2=O</smiles>

Stereochenol A<smiles>CC1CC2C=C3C(=O)c4ccccc4C(=O)[C@@]3(C)[C@@](C)(CO)C2C1</smiles>

Sterekunthal B

\section{Stereochenol B}<smiles>CC1=C2C=C3C(=O)c4ccccc4C(=O)C3(O)C(C)=C2CC1</smiles>

Sterequinone C
Saponins from Eclipta prostrata L. (Asteraceae) (Rahman et al., 2006; Rahman and Rashid, 2008)<smiles>[R20]OC(=C)C12CCC(C)(C)CC1C1=CCC3C4(C)CCC([R20])C(C)(C)C4CCC3(C)C1(C)CC2C</smiles>

$\mathrm{R}_{1}=\beta$-D-glucose, $\mathrm{R}_{2}=\mathrm{H}$ : Eclalbasaponin $\mathrm{I} ; \mathrm{R}_{1}=\mathrm{R}_{2}$ $=\beta$-D-glucose; Eclalbasaponin II 
Glycosides from Pterospermum semisagittatum

Buch. (Sterculiaceae) (Khan et al., 2003)<smiles>CC1=CC(=O)CC(C)(C)/C1=C/CC(C)OCl</smiles>

(Z)-4-[3'-( $\beta$-D-Glucopyranosyloxy)-butyli-dene]3,5,5-trimethyl-2-cyclohexen-1-one<smiles>CC1=CC(=O)CC(C)(C)/C1=C\CC(C)OCl</smiles>

(E)-4-[3'-( $\beta$-D-Glucopyranosy-loxy)-butyli-dene]3,5,5-trimethyl-2-cyclohexen-1-one<smiles>CC1=CC(=O)CC(C)(C)C1(O)CCC(C)O</smiles>

(E)-4-Hydroxy-4-[3'-( $\beta$-D-glucopyranosyloxy)butylidene]-3,5,5-trimethyl-2-cyclohexen-1-one

Diterpenoid from Potamogeton nodosus Poir. (Potamogetonaceae) (Qais et al., 1998)

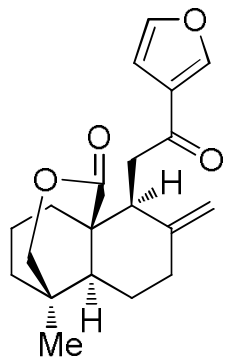

15,16-Epoxy-12-oxo-8(17),13(16),14-labdatrien20,19-olide

Diarylheptanoids from Garuga pinnata Roxb. (Burseraceae) (Ara et al., 2006)<smiles>[R]c1cccc(CCC(=O)/C=C(/CCc2ccc(O)c(-c3cc(CCC(=O)OC)ccc3[R])c2)OC)c1</smiles>

$\mathrm{R}=\mathrm{OH}$ : 6'-Hydroxygaruganin $\mathrm{V}$ $\mathrm{R}=\mathrm{H}$ : Garuganin V

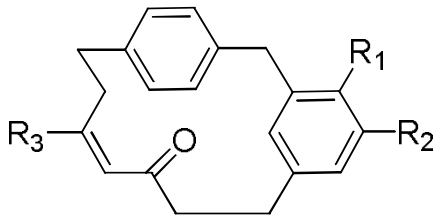

$\mathrm{R}_{1}=\mathrm{H}, \mathrm{R}_{2}=\mathrm{R}_{3}=\mathrm{OMe}$ : Garuganin IV

$\mathrm{R}_{1}=$ OMe, $\mathrm{R}_{2}=\mathrm{H}, \mathrm{R}_{3}=\mathrm{OH}:$ 9'- $^{\prime}$

Desmethylgarugamblin-I

$\mathrm{R}_{1}=\mathrm{R}_{2}=\mathrm{R}_{3}=$ OMe: Garuganin III

$\mathrm{R}_{1}=\mathrm{OH}, \mathrm{R}_{2}=\mathrm{R}_{3}=\mathrm{OMe}$ : 1-Desmethylgaruganin III

Triterpene and phenolics from Parmelia kamtschandlis Ach. (Parmeliaceae) (Mazid et al., 2001)<smiles>CC(=O)c1c(C)cc(O)c(C)c1O</smiles>

Methyl $\beta-$ orsellinate<smiles>CCOC(=O)c1c(C)cc(O)c(C=O)c1O</smiles>

Ethyl haemmatommate

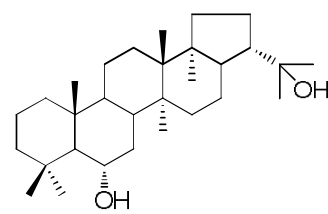

Hopane-6 $\alpha, 22$-diol<smiles>CCOC(=O)c1c(C)cc(O)cc1O</smiles>

Ethyl (6-methyl-2,4dihydroxy)-6-benzoate<smiles>CC(=O)C1=C(O)C2(C)C(=CC1=O)c1c(O)c(C)c(O)c(C)c1C(C(C)=O)=C2O</smiles>

Usnic acid

Flavonoids and triterpene from Erythrina variegata L. (Fabaceae) (Rahman et al., 2007)<smiles>CC1(C)C=Cc2c(cc3occ(-c4ccc(O)cc4)c(=O)c3c2O)O1</smiles>

Alpinum isoflavone<smiles>C=C(C)C1CCC2(C)CCC3(C)C(CCC4C5(C)CC[C@H](O)C(C)(C)C5CCC43C)C12</smiles>

Epilupeol 
<smiles>O=c1c(-c2ccc(O)cc2)coc2cc(O)c(O)c(O)c12</smiles>

6-Hydroxygenistein<smiles>CC1(C)CC(CO)C2CCC3(C)C(=CCC4C5CCC6C(C)(C)C(O)CCC6(C)C5CCC43C)C2C1</smiles>

3ß,28-Dihydroxyolean-12-ene<smiles>CC(C)=CCc1c(O)cc(O)c2c(=O)c(-c3ccc(O)cc3)coc12</smiles>

4',5,7-Trihydroxy-8-prenylisoflavones<smiles>Cc1c(O)cc(O)c2c(=O)c(-c3ccc(O)cc3)coc12</smiles>

4',5,7-Trihydroxy-8-methylisoflavone<smiles>CC(C)=CCc1c2c(c(O)c3c(=O)c(-c4ccc(O)cc4)coc13)C=CC(C)(C)O2</smiles>

Scandenone<smiles>CC(C)(O)CCc1c2c(c(O)c3c1OCC(c1ccc(O)cc1O)C3=O)C=CC(C)(C)O2</smiles>

2',4',5-Trihydroxyl-8- (3-methylbut-1(Z)enyl)-2",2"dimethylpyrano $\left[5^{\prime \prime}, 6^{\prime \prime}: 6,7\right]$ isoflavanone
Neolignans from Quisqualis indica $\mathbf{L}$. (Combretaceae) (Jahan et al., 2009)<smiles>[R]c1cc([C@@H](O)[C@H](C)Oc2c(OC)cc(C/C=C\C)cc2OC)cc(OC)c1[R]</smiles>

$\mathrm{R}=\mathrm{R}_{2}=\mathrm{H}, \mathrm{R}_{1}=\mathrm{OH}$ :

1-(4-Hydroxy-3-methoxyphenyl)-2-(4-allyl-2,6-

dimethoxyphenoxy)-propan-1-ol

$\mathrm{R}=\mathrm{R}_{2}=\mathrm{H}, \mathrm{R}_{1}=\mathrm{OMe}$ :

1-(3,4-Dimethoxyphenyl)-2-(4-allyl-2,6dimethoxyphenoxy)-propan-1-ol $\mathrm{R}=\mathrm{COCH}_{3}, \mathrm{R}_{1}=\mathrm{OMe}, \mathrm{R}_{2}=\mathrm{H}$ :

1-(3,4-dimethoxyphenyl)-2-(4-allyl-2,6-

dimethoxyphenoxy)-propan-1-ylacetate

$\mathrm{R}=\mathrm{H}, \mathrm{R}_{1}=\mathrm{OH}, \mathrm{R}_{2}=\mathrm{OMe}$ :

1-(4-Hydroxy-3,5-dimethoxyphenyl)-2-(4-allyl-2,6dimethoxyphenoxy)-propan-1-ol

Terpenoids Xanthium strumarium L. (Compositae) (Islam et al., 2009)<smiles>CC(=O)/C=C\C1=CC(O)C2C(CC1C)OC(=O)C2(O)O</smiles>

11-Hydroxy-11-carboxy-4-oxo-1(5), 2(Z)-xanthadien-12, 8-olide<smiles>COc1ccc(C(=O)OC2C=C(C)CCC3(O)C(C(C)C)CCC23C)cc1</smiles>

Lasidiol-10-anisate<smiles>CCC(CC[C@H](C)C1CCC2C3CC=C4C[C@@H](OC5OC(CO)C(O)C(O)C5(O)O)CC[C@]4(C)C3CC[C@@]21C)C(C)C</smiles>

Daucosterol 
Polyphenolics from Podocarpus neriifolius D.

(Podocarpaceae) (Rumzhum, 2008)

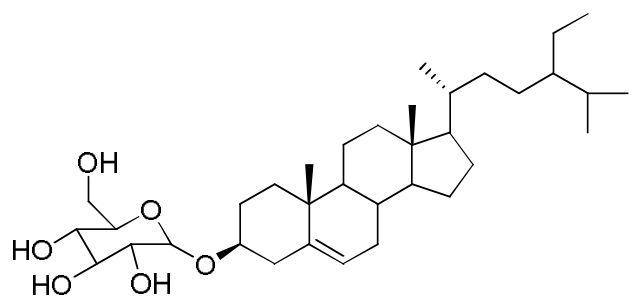

Daucosterol<smiles>CCOC(=O)c1cc(O)c(O)c(O)c1</smiles>

Ethyl gallate<smiles>COc1ccc(-c2cc(=O)c3c(O)cc(O)c(-c4cc(C5CC(=O)c6c(O)cc(OC)cc6O5)ccc4OC)c3o2)cc1</smiles>

Amentoflavone-4',4"', 7- trimethyl ether

Terpenoids and coumarins from Jatropha podagrica Hook. (Euphorbiaceae) (Rumzhum et al., 2011)<smiles>[R]c1cc2ccc(=O)oc2c([R3])c1[R]</smiles>

$\mathrm{R}_{1}=\mathrm{R}_{2}=\mathrm{COH}_{3}, \mathrm{R}_{3}=\mathrm{OH}:$ Fraxidin

$\mathrm{R}_{1}=\mathrm{COH}_{3}, \mathrm{R}_{2}=\mathrm{R}_{3}=\mathrm{OH}:$ Fraxetin

$\mathrm{R}_{1}=\mathrm{R}_{2}=\mathrm{COH}_{3}, \mathrm{R}_{3}=\mathrm{H}$ : Scoparone

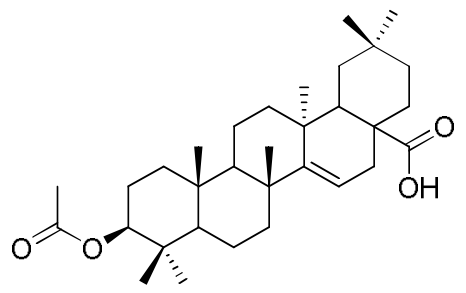

Acetylaleuritolic acid
Phenylpropanoid and Triterpene from Albizzia lebbeck L. (Leguminosae) (Hussain et al., 2008), Corypha taliera Roxb (Palmae) (Chowdhury et al.,

2013), Albizia chinensis (Osbeck.) Merr.

(Fabaceae) (Sharmin et al., 2013) and Mesua nagassarium Burm.f. (Clusiaceae) (Islam, 2012)<smiles>[R2]c1ccc(/C=C/C(=O)O)cc1[R]</smiles>

$\mathrm{R}_{1}=\mathrm{COH}_{3}, \mathrm{R}_{2}=\mathrm{OH}$ : Methoxycinnamic acid

$\mathrm{R}_{1}=\mathrm{H}, \mathrm{R}_{2}=\mathrm{OH}$ : Trans- $p$-coumaric acid<smiles>C=C(C)C1CCC2(C)CCCC3(C)C(CCC4C5(C)CCC(O)C(C)(C)C5CCC43C)C12</smiles>

Triterpenes from Couroupita guianensis Aubl. (Lecythidaceae), Corypha taliera Roxb (Palmae) (Chowdhury et al., 2013), Bryophyllum daigremontianum Raym. (Crassulaceae)

(Sharker et al., 2013) (Begum et al., 2009) and Glycosmis pentaphylla (Rutaceae) ( Ahmed, 2013)

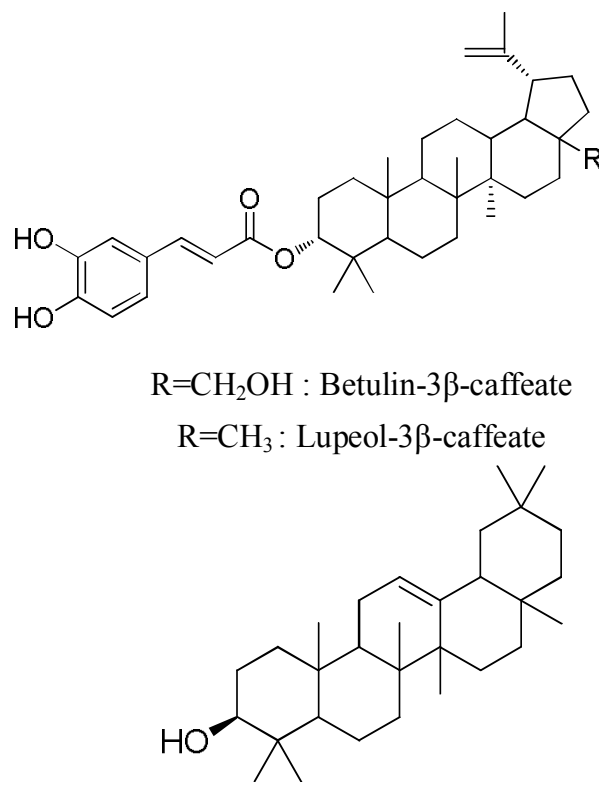

$$
\beta \text {-Amyrin }
$$


Unusual phenolic compounds from Streptomyces sp. (Jabbar et al., 1998)<smiles></smiles>

Dehyroaltenusinic acid<smiles></smiles>

Dehyroaltenusin<smiles>COc1cc(O)c(C(=O)O)c(-c2ccc(O)c(O)c2)c1</smiles>

Altenusin

Secondary metabolite from Monocillium sp.

(Biswas et al., 2000)<smiles>C=C1O[C@H]2[C@@H](NC1=O)C(C(=O)OC)=C[C@@H](O)[C@H]2O</smiles>

Monocillinol A<smiles>[CH][C@H]1C(C(=O)OC)=C[C@@H]2OC(=C)C(=O)N[C@@H]2[C@H]1O</smiles>

Monocillinol B
Triterpenes and flavonoid from Kalanchoe pinnata

(Lam.) (Crassulaceae) (Sharker et al., 2012), Corypha taliera Roxb (Palmae) (Chowdhury et al., 2013), Syzygium cumini L. (Murtaceae) (sikder $\boldsymbol{e t}$ al., 2012) and Mesua nagassarium Burm.f.

(Clusiaceae) (Islam, 2012)<smiles></smiles>

Glut-5(6)-en-3-one<smiles>CC1(C)CCC2(C)CC=C3C(C)(CCC4C3(C)CCC3C(C)(C)C(=O)CCC34C)C2C1</smiles>

Taraxerone<smiles>CC1[C@H](O)CCC2C34CCC5(C)C6CC(C)(C)CCC5(C)CCC6(C)C3CCC(C)(C4)C(C)C12C</smiles>

$\beta$-Friedelanol<smiles>CC(=O)O[C@H]1CCC2(C)C(CCC3(C)C2CC=C2C4CC(C)(C)CCC4(C)CCC23C)C1(C)C</smiles>

$\beta$-Amyrin-3-acetate<smiles>O=c1c(O)c(-c2ccc(O)c(O)c2)oc2cc(O)cc(O)c12</smiles>

3,5,7,3',5'-Pentahydroxyflavone

Triterpenes from Bryophyllum daigremontianum (Raym.) (Crassulaceae) (Sharker et al., 2013), Corypha taliera Roxb (Palmae) (Chowdhury et al., 2013) and Albizia chinensis (Osbeck.) Merr.

(Fabaceae) (Sharmin et al., 2013)<smiles></smiles>

Glut-5(6)-en-3 $\beta$-ol 
<smiles>CC[C@H](/C=C/[C@@H](C)[C@H]1CC[C@H]2[C@@H]3CC=C4CC(O)CC[C@]4(C)[C@H]3CC[C@@]21C)C(C)C</smiles>

Stigmasterol<smiles>CC1CCC2(C)CCC3(C)C(=CCC4C5(C)CCC(O)C(C)(C)C5CCC43C)C2C1C</smiles>

Secondary metabolites from Melocanna baccifera

Roxb. (Kuddus et al., 2011)<smiles>O=C(O)/C=C/C=C\c1ccc2c(c1)OCO2</smiles>

Isochavicinic acid<smiles>C=CCc1ccc(O)c(OC)c1</smiles>

Eugenol<smiles>CC(=O)O[C@]1(C)CC[C@@]2(C)C(CC[C@]3(C)C2CC=C2C4CC(C)(C)CC[C@]4(C(=O)O)CC[C@@]23C)C1(C)C</smiles>

Olean-12-en-28-carboxy-3 $\beta$-acetate

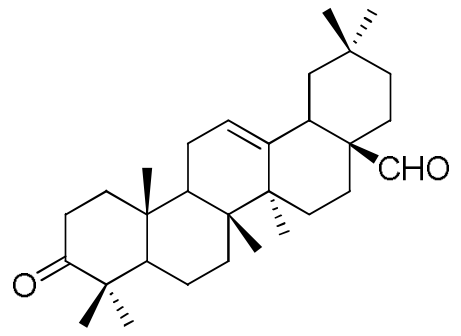

3-Oxo-olean-12-en-28-al

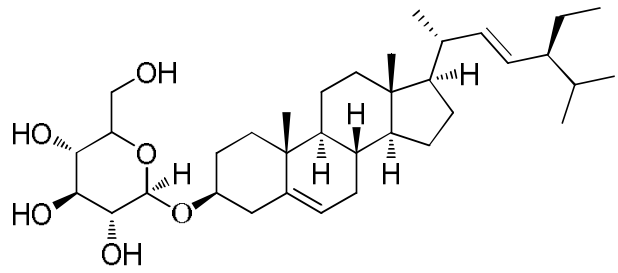

Stigmasterol glucoside<smiles></smiles>

$\beta$-Sitosterol glucoside

Sesquiterpene and Phenylpropanoids from Curcuma longa L. (Zingiberaceae) (Kuddus et al., 2010) and Syzygium cumini L. (Murtaceae) (Sikder et al., 2012)<smiles>CC(C)=CC(=O)CC(C)c1ccc(C)cc1</smiles>

Turmerone<smiles>COc1cc(/C=C/C(=O)O)ccc1O</smiles>

Trans-ferulic acid<smiles>Cc1cc2c(cc1O)C(C)CCC2C(C)C</smiles>

7-Hydroxycalamenene<smiles>O=C(O)/C=C/c1ccc(O)cc1</smiles>

Trans-p-coumaric acid<smiles>COc1ccc(/C=C/C(=O)O)cc1O</smiles>

Trans-isoferulic acid<smiles>COC(OC)c1cc(C)c(O)cc1C</smiles>

Methyl- $\beta$-orsellinate
Acridone-type alkaloid and triterpene from Glycosmis pentaphylla Retz. (Rutaceae) (Ahmed, 2013) and Mesua nagassarium Burm.f.

(Clusiaceae) (Islam, 2012)<smiles>CCC(/C=C/[C@H](C)C1CCC2C3=CCC4C[C@@H](O)CC[C@]4(C)C3CC[C@]21C)C(C)C</smiles>

Spinasterol<smiles>CC1(C)CCC2(C)CCC3(C)C(=CCC4C5(C)CC[C@@H](O)C(C)(C)C5CCC43C)C2C1</smiles>

Epi-oleanolic acid 
<smiles>COc1cc2c(c(O)c1OC)c(=O)c1ccccc1n2C</smiles>

Arborinine

Constituents from Ophiorrhiza mungos Linn.

(Rubiaceae) (Islam, 2010)<smiles>CC1=CC(=O)CC(C)(C)[C@@]1(O)/C=C/C(C)O</smiles>

Vomifoliol<smiles>COc1cc2ccc(=O)oc2cc1O</smiles>

Scopoletin.
1-Methyl-7-methoxy- $\beta$-carboline<smiles>O=C(O)CCc1nccc2c1[nH]c1cc(O)ccc12</smiles>

7-Hydroxy-beta carboline-1-propionic acid

Biological:

i) Antimicrobial activity:

Table 2. Antimicrobial activity of Garuganin V from Garuga pinnata at $100 \mu \mathrm{g} / \mathrm{disc}$ (Ara et al., 2012).

\begin{tabular}{lcc}
\hline \multirow{2}{*}{ Microbes } & \multicolumn{2}{c}{ Diameter of zone of inhibition (mm) } \\
\cline { 2 - 3 } Gram positive bacteria & Garuganin $\mathrm{V}$ & Structure (sample) \\
Bacillus cereus & 40 & 21 \\
Staphylococcus aureus & 35 & 23 \\
Gram negative bacteria & 32 & 21 \\
Escherichia coli & 36 & 20 \\
aVibrio mimicus & 31 & 20 \\
Fungus & 35 & \\
Aspergillus niger & & \\
Candida albicans & &
\end{tabular}

Table 3. Comparative antibacterial activity of usnic acid and standard antibiotics (Rashid et al., 2001).

\begin{tabular}{lcccc}
\hline \multirow{2}{*}{ Microbes } & \multicolumn{5}{c}{ Diameter of zone of inhibition (mm) } \\
\cline { 2 - 6 } & Usnic acid & Amoxycillin & Streptomycin & Tetracycline \\
\cline { 2 - 6 } & $30 \mu \mathrm{g} / \mathrm{disc}$ & $10 \mu \mathrm{g} / \mathrm{disc}$ & $10 \mu \mathrm{g} / \mathrm{disc}$ & $30 \mu \mathrm{g} / \mathrm{disc}$ \\
\hline Bacillus subtilis & 23 & 33 & 26 & 34 \\
Escherichia coli & 25 & 08 & 22 & 18 \\
Staphylococcus aureus & 24 & - & & \\
Stap. epidermidis & 23 & 39 & &
\end{tabular}


ii) Antioxidant Activity

Table 4. Free radical scavenging of some purified compounds (Begum et al., 2009; Begum et al., 2011).

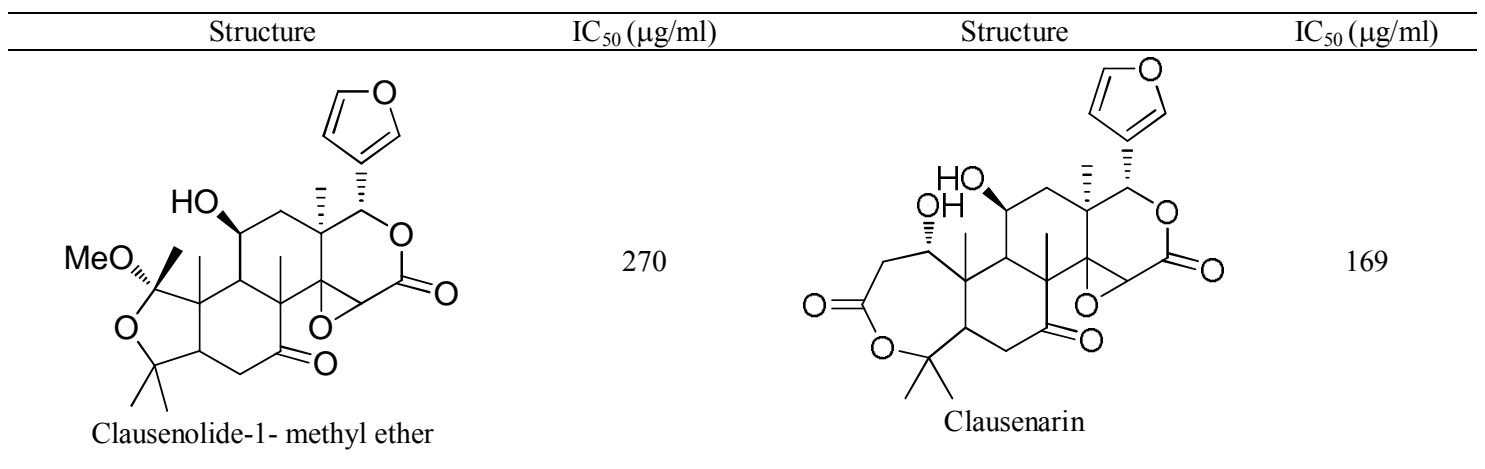

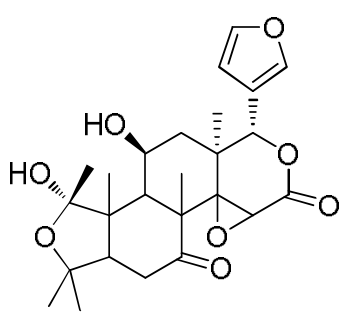

Clausenolide
102<smiles>CC(C)=CCc1c(O)c(C=O)cc2c1[nH]c1c(O)cccc12</smiles>

8-Hydroxyheptaphylline
106

$? ?$ iii) Antidiabetic activity: The glucose level obtained in the blood of normal and experimental rats are given in table 5 for E. prostrata extract and in table 6 for eclalbasaponin II. The safety of the extractives in animal was evaluated by observing the effects of the extractives on liver enzymes. The levels of enzymes, alanine aminotransferase (ALT), aspartate aminotransferase (AST) and alkaline phosphatase (ALP) in plasma of normal and diabetic rats are depicted in table- 8 . The plant extract treated animals showed no significant changes of these enzyme levels as compared to the normal rats. This suggested that the extractives were safe in rat models. However, the levels of these enzymes were much lower than the diabetic control rats receiving no drugs.

The methanolic extract $(300 \mathrm{mg} / \mathrm{kg})$ showed a significant $\quad(\mathrm{P}<0.05)$ blood glucose reduction $(14.50 \%)$ on 7 th day in diabetic rats as compared to the untreated diabetic rats (Table 6). Consequently, serum sugar reducing activity became significant $(\mathrm{P}<0.001)$ after $21(43.18 \%)$ and 28 days $(48.38 \%)$ of drug treatment. The purified compound, eclalbasaponin II $(10 \mathrm{mg} / \mathrm{kg})$ also reduced the serum sugar level $(16.07 \%)$ significantly $(\mathrm{P}<0.001)$ after 3 days of treatment when compared with the untreated diabetic rats. The blood sugar lowering effects were increased after $5(36.53 \%)$ and 7 days $(52.90 \%)$ by eclalbasaponin II. In alloxan-induced diabetic rats the levels of plasma AST, ALT and ALP were significantly $(\mathrm{P}<0.001)$ increased by $93.48 \%, 64.30 \%$ and $81.44 \%$, respectively relative to their normal levels in rats (Table 8). On the other hand, treatment of the diabetic rats with methanolic extract of the $E$. prostrata caused a reduction in the activity $43.74 \%$, $37.97 \%$ and $48.09 \%$ of ALT, AST and ALP in blood plasma as compared to the mean values in the diabetic rats. It was also observed that there was no significant difference in the liver enzyme levels between the normal, glibenclamide and E. prostrata 
treated rats. Therefore, the herb did not have any hepatotoxicity on rats (Rahman and Rashid, 2008). Similar hypoglycemic activity has been reported for glycoside D ( $\beta$-D-galactopyranosyl) from Calendula officinalis (Fam.- Compositae) (Yoshikawa et al.,
2001). Glycoside D and eclalbasaponin II are structurally related, both of which have been obtained from the member of the same family.

Table 5. Blood sugar levels in normal and alloxan - induced diabetic rats (Phase I) (Rahman et al., 2011).

\begin{tabular}{lccccc}
\hline Groups & \multicolumn{4}{c}{$\mathrm{mmol} / \mathrm{l}$} \\
\cline { 2 - 6 } & 1st day & 7th day & 14th day & 21st day & 28th day \\
\hline Normal (untreated) & $4.85 \pm 0.08$ & $5.02 \pm 0.10$ & $4.91 \pm 0.07$ & $4.79 \pm 0.11$ & $4.85 \pm 0.06$ \\
\hline Diabetic control & $12.03 \pm 0.18^{* *}$ & $12.98 \pm 0.19^{* *}$ & $14.05 \pm 0.23^{* *}$ & $15.09 \pm 0.28^{* *}$ & $17.20 \pm 0.22^{* *}$ \\
\hline Glibenclamide Treated $(1 \mathrm{mg} / \mathrm{kg} \mathrm{bw})$ & $12.18 \pm 0.55$ & $10.82 \pm 0.18$ & $9.53 \pm 0.21$ & $7.08 \pm 0.16$ & $6.43 \pm 0.16$ \\
\hline $\begin{array}{l}\text { Methanolic extract Treated } \\
(300 \mathrm{mg} / \mathrm{kg} \mathrm{bw})\end{array}$ & $12.69 \pm 0.32$ & $10.85 \pm 0.16^{*}$ & $9.37 \pm 0.20^{* *}$ & $7.21 \pm 0.24^{* *}$ & $6.55 \pm 0.11^{* *}$
\end{tabular}

Values are given as mean \pm SEM for 6 rats in each group. Diabetic control (Group-2) was compared with normal (Group-1) on corresponding day. Experimental group (Group-4) was compared with diabetic control group on corresponding day; $* \mathrm{P}<0.05 ; * * \mathrm{P}<0.001$

Table 6. Blood sugar level in normal and alloxan - induced diabetic rats (Phase II) (Rahman et al., 2011).

\begin{tabular}{lcccc}
\hline \multirow{2}{*}{ Groups } & \multicolumn{4}{c}{$\mathrm{mmol} / \mathrm{L}$} \\
\cline { 2 - 5 } & 1st day & 3rd day & 5th day & 7th day \\
\hline Normal untreated & $4.8 \pm 0.56$ & $5.03 \pm 0.48$ & $4.85 \pm 0.55$ & $4.95 \pm 0.40$ \\
Diabetic control & $12.40 \pm 0.35^{* *}$ & $12.49 \pm 0.44^{* *}$ & $12.97 \pm 0.51^{* *}$ & $13.52 \pm 0.34^{* *}$ \\
Glibenclamide treated $(1 \mathrm{mg} / \mathrm{kg} \mathrm{bw})$ & $12.78 \pm 0.25$ & $12.01 \pm 0.31$ & $11.63 \pm 0.26$ & $10.51 \pm 0.35$ \\
Eclalbasaponin II treated $(10 \mathrm{mg} / \mathrm{kg} \mathrm{bw})$ & $12.87 \pm 0.68$ & $10.80 \pm 0.71^{* *}$ & $8.17 \pm 0.65^{* *}$ & $6.06 \pm 0.66^{* *}$ \\
\hline
\end{tabular}

Values are given as mean \pm SEM for 6 rats in each group. Diabetic control (Group-2) was compared with normal (Group-1) on corresponding day; Experimental group (Group-4) was compared with diabetic control group on corresponding day; $* \mathrm{P}<0.05 ; * * \mathrm{P}<0.001$

Table 7. Percentage reduction of blood sugar level in alloxan induced diabetes rats.

\begin{tabular}{cccc}
\hline Day & \% Reduction of blood sugar & Day & \multicolumn{2}{c}{ \% Reduction of blood sugar } \\
\cline { 2 - 2 } & Crude extract & & Eclalbasaponin II \\
\hline 1 & 0.000 & 1 & 0.000 \\
7 & 14.50 & 3 & 16.07 \\
14 & 26.16 & 5 & 36.53 \\
21 & 43.18 & 7 & 52.90 \\
28 & 48.38 & - & - \\
\hline
\end{tabular}

Table 8. ALT, AST and ALP levels of normal and alloxan-induced diabetic rats after 4 weeks.

\begin{tabular}{lccc}
\hline Groups & \multicolumn{3}{c}{ U/L } \\
\cline { 2 - 4 } & ALT & AST & ALP \\
\hline Normal untreated & $28.34 \pm 0.88$ & $166.66 \pm 2.15$ & $60.16 \pm 3.39$ \\
Diabetic control & $54.5 \pm 2.70^{* *}$ & $273.83 \pm 3.37^{* *}$ & $109.16 \pm 1.93^{* *}$ \\
Glibenclamide treated & $31.83 \pm 2.77$ & $196.16 \pm 3.51$ & $58.83 \pm 3.26$ \\
Methanolic extract treated & $30.66 \pm 1.33^{* *}$ & $169.83 \pm 3.85^{* *}$ & $56.66 \pm 3.84^{* *}$ \\
\hline
\end{tabular}

Values are given as mean \pm SEM for 6 rats in each group. Diabetic control (Group-2) was compared with normal (Group-1). Experimental group (Group-4) was compared with diabetic control (Group-2). $* * \mathrm{P}<0.001$ 
Anti-HIV activity: The anti-HIV activity of dehydroaltenusin from Streptomyces sp. (Jabbar et al., 1999 is shown below). Dehydroaltenusin revealed significant anti HIV activity,

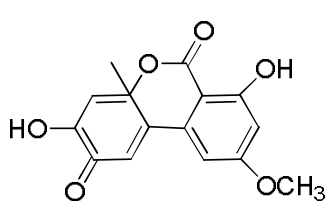

Dehydroaltenusin

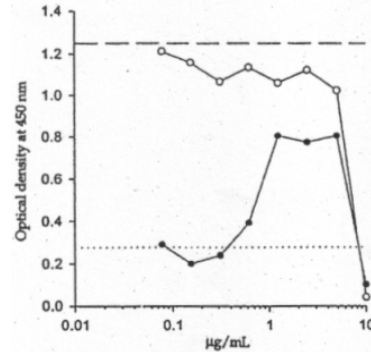

Figure 1. Graph showing the effects of dehydroaltenusin upon uninfected CEM-SS (o) and HIV-1 infected CEM-SS cells $(\bullet)$, as determinded after 6 days of culture. The higher optical density represents better anti HIV activity exhibited by the test compound

\section{Conclusion}

A total of 60 plant species have been investigated. Many structurally unique and diversified compounds having interesting biological activities were isolated from these plants. Our studies show that Bangladeshi plants can be a promising source of novel drug candidates.

\section{References}

Ahamed, B.M.K., Krishna, V., Gowdru, H.B., Rajanaika, H., Kumaraswamy, H.M., Rajshekarappa, S., Dandin, C.J. and Mahadevan, K.M. 2007. Isolation of bactericidal constituents from the stem bark extracts of Grewia tiliaefolia Vahl. Res. J. Med. Plant. 1, 7282.

Aher, A.N., Pal. S.C., Yadav, S.K., Patil, U.K. and Bhattacharya, S. 2009. Antioxidant activity of isolated phytoconstituents from Casuarina equisetifolia Frost (Casuarinaceae). J. Plant Sci. 4, 15-20.

Ahmed, I. 2013. Chemical and biological studies of Glycosmis pentaphylla (Retz.) DC and Polyalthia longifolia Sonn. var. pendulla. M. Pharm Thesis, University of Dhaka.

Ara, K, Kaisar, M.A., Rahman, M.S., Chowdhury, S.R., Islam, F. and Rashid, M.A. 2012. Antimicrobial Constituents from Garuga pinnata Roxb. Lat. Am. J. Pharm. 31, 1071-1073.

Ara, K., Rahman, A.H.M.M., Hasan, C.M., Iskander, M.N., Asakawa, Y., Quang, D.N. and Rashid, M.A. 2006. Macrocyclic diarylheptanoids from Garuga pinnata. Phytochemistry 67, 2659-2662.

Asha, K.M., Chowdhury, R., Hasan, C.M., and Rashid, M.A. 2003. Antibacterial activity and cytotoxicity of extractives from Unona discolor/ Uvaria hamiltonii stem bark. Fitoterapia 74, 159-163.

Begum R., Rahman M.S., Chowdhury A.M.S., Rahman M.M. and Rashid M.A. 2008. $O$-methylheptaphylline from Clausena suffruticosa. Nat. Prod. Commun. 3, $1-4$.

Begum, R., Kaisar, M.A., Rahman, M.S., Chowdhury, A.M.S., Rahman, M.M., Hasan, C.M. and Rashid, M.A. 2009. Clausenolide-1-methyl ether from Clausena heptaphylla W\&A. Bol. Latinoam. Caribe. Plant Med. Aromat. 10, 136-138.

Begum, R., Rahman, M.S., Chowdhury, A.M.S., Hasan, C.M. and Rashid, M.A. 2009. Secondary metabolites (Triterpenes) from Couroupita guianensis. Ori. Pharm. Exper. Med. 9, 200-205.

Begum, R., Rashid, M.A. and Chowdhury, A.M.S. 2011. Medicinal Plants of Bangladesh, Volume-I: Chemical Constituents and Bioactivities. LAMBERT Academic Publishing, Germany.

Bhilabutra, W., Techowisan, T., Peberdy, J.F. and Lumyong, S. 2007. Antimicrobial activity of bioactive compounds from Periconia siamensis CMUGE015. Res. J. Microbiol. 2, 749-755.

Biswas, M. H.U., Amin, A.R.M.R., Islam, M.A., Hasan, C.M., Gustafson, K.R., Boyd, M.R., Pennell, L.K. and Rashid, M.A. 2000. Monocillinols A \& B, novel fungal metabolites from a Monocillium sp. Tetrahedron Lett. 41, 7177-7180.

Brand-Williams, W., Cuvelier, M.E. and Berset, C. 1995. Use of a free radical method to evaluate antioxidant activity. Food Sci. Tech. 28, 25-30.

Burnett, J., Newman, B. and Sun, D. 2012. Targeting cancer stem cells with natural products. Curr. Drug Targets. 13, 1054-1064.

Chowdhury, R., Hasan, C.M. and Rashid M.A. 2003. Guaiane sesquiterpenes from Amoora rohituka. Phytochemistry 62, 1213-1216.

Chowdhury, R., Rashid, R.B., Sohrab M.H., Hasan C.M. 2003. 12 $\alpha$-hydroxystigmast-4-en-3-one: a new bioactive steroid from Toona ciliata (Meliaceae). Pharmazie 58, 272-273.

Chowdhury, A., Alam, M.A., Rashid, R.B., Al-Mansur, M.A., Rahman M.S. and Rashid, M.A. 2013. Steroids and triterpenoids from Corypha taliera Roxb: a critically endangered palm species of Bangladesh. Res. J. Med. Plant 7, 125-129.

Christen, P. and Cuendet, M. 2012. Plants as a source of therapeutic and health products. Chimia (Aarau) 66, 320-323.

Connolly J.D., Haque M.D.E.,, Hasan C.M. and Hossain M.S. 1994. 15a-hydroxy-24-methylenelanosta-7,9(11)dien-3-one from the Stem bark of Desmos longiflorus. Phytochemistry 36, 1337-1338. 
Datta, B.K., Datta, S.K., Rashid, M.A., Kundu, J.K., Hasan, C.M. and Sarker, S.D. 2002. Furhter sequiterpenes from Polygonum viscosum (Polygonaceae). Nat. Prod. Lett. 16, 143-148.

Farruque, R., Chowdhury, R., Sohrab, M.H., Hasan, C.M. and Rashid, M.A. 2003. Triterpene constituents from the leaves of Melicope indica. Pharmazie 58, 518-520.

Ghani, N.A., Ahmat, N., Ismail, N.H., Zakaria, I. and Zawawi, N.K.N. A. 2012. Chemical constituents and cytotoxic activity of Polyalthia cauliflora var. cauliflora. Res. J. Med. Plant. 6, 74-82.

Gulakowski, R.J., McMahon, J.B., Staley, P.G. Moran, R.A. and Boyd, M.R. 1991. A semiautomated multiparameter approach for anti-HIV drug screening. J. Virol. Methods 33, 87-100.

Ham, Y.M., Kim, K.N., Lee, W.J., Lee, N.H. and Hyun, C.G. 2010. Anti-inflammatory effects of apo-9'fucoxanthinone from the brown alga, Sargassum muticum. Int. J. Pharmacol. 6, 147-151

Haque, M.R., Rahman, K.M., Iskander, M.N., Hasan, C.M. and Rashid M.A. 2006. Stereochenols A and B, two quinones from Stereospermum chelonoides. Phytochemistry 67, 2663-2665.

Hasan, C.M., Hossain, M.A. and Rashid, M.A. 1995. Clerodane diterpenoids from Polyalthia longifolia var. pendulla. Biochem. Syst. Ecol. 23, 331-332.

Hasan, C.M., Huda, Q., Lavaud C., Connolly, J.D. and Huq, M.E. 1995. Bengalensol, a new 16-epicafestol derivative from the leaves of Coffea bengalensis. Nat. Prod. Lett. 4, 55-60.

Hasan, C.M., Islam, A., Ahmed, M., Ahmed, M.U. and Waterman, P.G. 1984. Capsugenin, a dammarane triterpene from Corchorus capsularis. Phytochemistry 23, 2583-2587.

Hasan, C.M., Khan, S., Jabbar, A. and Rashid, M.A. 2000. Two novel neoclerodane diterpenes from Barringtonia recemosa. J. Nat. Prod. 63, 411-412.

Hasan, C.M., Shahanz, S., Ilias, M., Gray, A.I. and Waterman, P.G. 1987. Chemistry in the Annonaceae, XXIII. 24-Methylene-lanosta-7,9(11)-dien-3 $\beta$-ol from Artabotrys odorotissimus stem bark. J. Nat. Prod. 50, 762-763.

Hasan, C.M., Mia, M.Y. and Rashid, M.A. 1996. A new acetogenin from Goniothalamus sesquipedalis (Annonaceae). Dhaka Univ. J. Biol. Sci. 5, 99-102.

Hasan, C.M., Mia, M.Y., Rashid, M.A. and Connolly, J.D. 1994. 5-Acetoxyisogoniothalamin oxide, A new epoxystyryl lactone from Goniothalamus sesquipedalis (Annonaceae). Phytochemistry 37, 17631764.

Hung, H.Y., Qian, K., Morris-Natschke, S.L., Hsu, C.S. and Lee, K.H. 2012. Recent discovery of plant-derived anti-diabetic natural products. Nat. Prod. Rep. 29, 580606.

Huq, M.M., Jabbar, A., Rashid, M.A., Hasan, C.M. 1999 b. A novel antibacterial and cardiac steroid from the roots of Nerium oleander. Fitoterapia 70, 5-9.
Huq, M.M., Jabbar, A., Rashid, M.A., Hasan, C.M., Ito, C. and Furukawa, H. 1999a. Steroids from the roots of Nerium oleander. J. Nat. Prod. 62, 1055-1057.

Hussain, M.M, Rahman, M.S. Jabbar, A. and Rashid, M.A. 2008 Phytochemical and biological investigations of Albizzia lebbeck Benth. Lat. Am. Carib. Bull. Med. Arom. Plants 8, 273-278.

Islam, F. 2010. Chemical and biological investigations of Ophiorrhiza mungos (L) and Mussaenda macrophylla (Wall). M. Pharm Thesis, University of Dhaka.

Islam, R. 2012. Chemical and biological investigations of Mesua nagassarium (Burm.f.) and Polyalthia longifolia Sonn. var. pendulla. M. Pharm Thesis, University of Dhaka.

Islam, M.R., Uddin, M.Z., Rahman, M.S., Tutul, E., Rahman, M.Z., Hassan, M.A., Faiz, M.A., Hossain, M., Hussain, M. and Rashid, M.A. 2009. Ethnobotanical, phytochemical and toxicological Studies of Xanthium strumarium L. Bang. Med. Res. Counc. Bull. 35, 84-90.

Jabbar, A., Shresta, A., Rashid, M.A., Shameem, M., and Yahara, S. 1998. Dehydroaltenusinic acid- a novel microbial metabolite from a Streptomyces sp. Nat. Prod. Lett. 12, 311-316.

Jabbar, A., Shresta, A.P., Hasan, C.M. and Rashid, M.A. 1999. Anti-HIV activity of dehydroaltenusin- a metaboite from a steptomyces sp. Nat. Prod. Sci. 5, 162-164.

Jahan, F.N., Rahman, M.S., Rahman, M.M., Gibbons, S., Masud, M.M., Sadhu, S.K., Hossain, M., Hasan, C.M. and Rashid, M.A. 2009. Diphenylpropanoids from Quisqualis indica and their anti-staphylococcal activity. Lat. Am. J. Pharm. 28, 279-83.

Jain, P.S. and Bari, S.B. 2010. Isolation of lupeol, stigmasterol and campesterol from petroleum ether extract of woody stem of Wrightia tinctoria. Asian J. Plant Sci. 9, 163 - 167.

Jumana, S., Hasan, C.M. and Rashid, M.A. 2000a. (+)Isocorydine- $\alpha-N$-oxide : A new aporphine alkaloid from M. velutina. Nat. Prod. Lett. 14, 393-397.

Jumana, S., Hasan, C.M. and Rashid, M.A. 2000 b. Antibacterial activity and cytotoxicity of Miliusa velutina. Fitoterapia 71, 559-561.

Khan, M.S.H., Nahar, N., Mosihuzzaman ,M., and Rashid, M.A. 2003. Three megastigmane glycosides from the leaves of Pterospermum semisagittatum. Pharm. Biol. 41, 11-15.

Kuddus, M.R., Rumi, F., Kaisar, M.A. and Hasan, C.M. 2010. Sesquiterpene and phenylpropanoids from Curcuma longa. Bang. Pharm. J. 13, 31-34.

Kuddus, M.R., Rumi, F., Kaisar, M.A., Hasan, C.M., Hassan, and Rashid, M.A. 2010. Trans-isoferulic acid from Curcuma longa. Bol. Latinoam. Caribe Plant. Med. Aromat. 9, 319-321. 
Kuddus, M.R., Rumi, F., Kaisar, M.A., Rahman, M.S., Hasan, C.M., Hassan, and Rashid, M.A. 2011. Secondary metabolites from Melocanna Baccifera (Roxb.). Asian J. Chem. 23, 85-88.

Lovkova, M.Y., Buzuk, G.N., Sokolova, S.M. and Kliment'eva, N.I. 2001. Chemical features of Medicinal Plants (Review). Appl. Biochem. Microbiol. 37, 229-237.

Mansour H.A., Newairy A.A., Yousef M.I. and Sheweita S.A. 2002. Biochemical study on the effects of some Egyptian herbs in alloxan-induced diabetic rats. Toxicology 170, 221-228.

Mazid, M.A., Sohrab, M.H., Hasan, C.M. and Rashid M.A. 2001. Chemical and biological studies of Parmelia kamtschandalis Ach. Dhaka Univ. J. Biol. Sci. 10, 6978.

Moustafa, A.M.Y., Kouam, S.F., Kulsoom, A., Ejaz, A., Ali, S., Anjum, S. and Choudhary, M.I. 2007. Phytochemical investigation biological evaluation of Schinus terebinthifolius. Res. J. Phytochem. 1, 1-11.

Newman, D.J. and Cragg, G.M. 2012. Natural products as sources of new drugs over the 30 years from 1981 to 2010. J. Nat. Prod. 75, 311-335.

Nutun, M.T.H., Hasan, C.M. and Rashid, M.A. Bismurrayafoline E. 1999. A new dimeric carbazole alkaloid from Murraya koenigii. Fitoterapia 70, 130-133.

Qais, N., Mandal, M.R., Rashid, M.A., Jabbar, A., Koshino, H., Nakata, T.NT. 1998. A furanoid labdane diterpene from Potamogeton nodusus. J. Nat. Prod. 61, 156-157.

Quader, M.A., Ahmed, M.U., Hasan, C.M. and Waterman, P.G. 1987. A new glycoside from the leaves of Corchorus capsularies. J. Nat. Prod. 50, 479-481.

Rahman, M.S., Chowdhury, R., Begum, B., Rahman, K.M. and Rashid, M.A. 2005. Phytochemical studies of Amoora cucullata. Dhaka Univ. J. of Pharm. Sci. 4, 73-75.

Rahman, S., Hasnat, A., Hasan, C.M., Rashid, M.A. and Ilias, M. 2001. Pharmacological evaluation of Bangladeshi medicinal plants - a review. Pharm. Biol. 39, 1-6.

Rahman, M.S. and Rashid, M.A. 2008. Antimicrobial activity and cytotoxicity of Eclipta prostrata. Ori. Pharm. Exp. Med. 8, 47-52

Rahman, M.S., . Rahman, M.Z, Begum, B., Chowdhury, R., Islam, S.N. and Rashid, M.A. 2011. Antidiabetic principle from Eclipta prostrata. Lat. Am. J. Pharm. 30, $1656-1660$.

Rahman, M.S., Chowdhury, R., Hasan, C.M. and Rashid, M.A. 2006. Oleanane Glycosides from Eclipta prostrata. Dhaka Univ. J. Pharm. Sci. 4, 107-111.

Rahman, M.Z., Sultana, S.J., Faroquee, C.F., Ferdous, F., Rahman, M.S., Islam, M.S. and Rashid, M.A. 2007. Phytochemical and biological investigations of Erythrina variegata. Saudi Pharm. J. 15, 140-145.
Rashid, M.A., Bhuiyan, M.S.A., Hossain, M.A., Quader, M.A., Nutan, M.T.H. and Karim, M.R. 2001. (+)Usnic acid, an antimicrobial agent from Permelia kamtschandalis. Pak. Jour. Mic. 1, 26-28.

Rumzhum, N.N. 2008. Chemical and Biological Investigations of Podocarpus neriifolius. M. Pharm Thesis, University of Dhaka.

Rumzhum, N.N., Sohrabb, M.H., Al-Mansurb, M.A., Hasan, C.M., Rashid, M.A. 2011. Secondary metabolites from Jatropha podagrica Hook. J. Phy. Sci. 23, 29 - 37.

Sharker, S.M., Hossain, M.K., Haque, M.R., Chowdhury, A.A., Kaisar, M.A. Hasan, C.M. and Rashid, M.A. 2012. Chemical and biological studies of Kalanchoe pinnata (Lam.) growing in Bangladesh. Asian Pacific J. Trop. Biomed. S1317-S1322.

Sharker, S.M., Hossain, M.K., Haque, M.R., Kabir, A.N.M.H., Hasan, C.M. and Rashid, M.A. 2013. Phytochemical and pharmacological studies of Bryophyllum daigremontianum (Raym.). Am. J. Pharm. Tech. Res. 3, 484 - 492.

Sharmin, T., Islam, F., Kaisar, M.A., Mansur, M.A., Sikder, M.A. and Rashid M.A. 2013. Chemical and biological investigations of Albizia chinensis (Osbeck.) Merr. $J$. Phy. Sci. (Accepted)

Sikder, M.A., Kaisar, M.A., Rahman, M.S., Hasan, C.M., Rehaily, J.A. and Rashid, M.A. 2012. Secondary metabolites from seed extracts of Syzygium cumini (L.). J. Phy. Sci. 23, 83-87.

Sohrab, M.H., Chowdhury, R., Hasan, C.M., and Rashid, M.A. 2004. Chemotaxonomic significance of polyoxygenated flavonoids from the leaves of Micromelum minutum. Biochem. Syst. Ecol. 32, 829831.

Sohrab, M.H., Hasan, C.M., and Rashid, M.A. 1999. Lunamarins A and B: two novel terpenoid coumarins from Clausena heptaphylla. Nat. Prod. Lett. 14, 47-53.

Sunilson, J.A.J., Suraj, R., Rejitha, G., Anandarajagopal, K., Kumari, A.V.A.G. and Promwichit, P. 2009. In vitro antimicrobial evaluation of Zingiber officinale, Curcuma longa and Alpinia galanga extracts as natural food preservatives. Am. J. Food. Tech. 4, 192200.

Widodo, G.P., Sukanda,r E., Sukrasno, Y. and Adnyana, I.K. 2008. A coumarin from Ageratum leaves (Ageratum conyzoides L.). Int. J. Pharmacol. 4, 5659.

Yoshikawa, M., Murakami, T., Kishi, A., Kageura, T. and Matsuda, H. 2001. Medicinal flowers. III. marigold. (1): hypoglycemic, gastric emptying inhibitory, and gastroprotective principles and new oleanane-type triterpene oligoglycosides, Calendasaponins A, B, C, and D from Egyptian Calendula officinalis. Chem. Pharm. Bull. 49, 863-870.

Zhu, H., Fu, P. and Zou, D.F. 2003. Chemical constituents of petroleum ether extract from Nervilia foadii. Pak. J. Biol. Sci. 9, 1556-1558. 
